International Journal of Biomedicine I June 2019 - Volume 9, Issue Suppl_1: Abstracts From the Second Russian International Conference "Cryo-electron microscopy 2019: achievements and prospects"

ORAL ABSTRACT PRESENTATIONS

SESSION TITLE: MOLECULAR ORGANIZATION OF CELLS AND ORGANELLES

DOI: 10.21103/IJBM.9.Suppl_1.OR6

\title{
Abstract OR-6: Looking for an Order in Chaos: Approaches to Mapping Chromosomal Loci for Cryo-EM Studies
}

Igor I.Kireev, Amir N.Zakirov, Sophia Yu. Sosnovskaya, Olga S.Strelkova, Oxana A. Zhironkina

A.N.Belozersky Institute of Physico-chemical biology, Lomonosov Moscow State University, Moscow, Russia

Background: Chromatin higher order structure has been for decades a matter of debate, mainly due to its mesoscale nature, which precluded its accurate analysis by optical microscopy, while electron microscopy studies rendered controversial results reflecting highly dynamic and heterogeneous DNA folding prone to sample preparation artifacts. On the other hand, lack of adequate tools for nondestructive yet selective staining of DNA as a whole, and specific chromosomal loci in particular, added another layer of uncertainty to the fuzzy picture of how DNA is organized in the cell nucleus.

Methods: Here we propose an approach for specific labeling of endogenous chromosomal loci compatible with the direct visualization of DNA folding motifs at near native conditions by cryo-electron tomography. The approach is based on use of tagged DNA-binding proteins for mapping chromosomal loci, with subsequent introduction of Nanogold-conjugated ligands into the cell nuclei by microinjection (Fig.1).

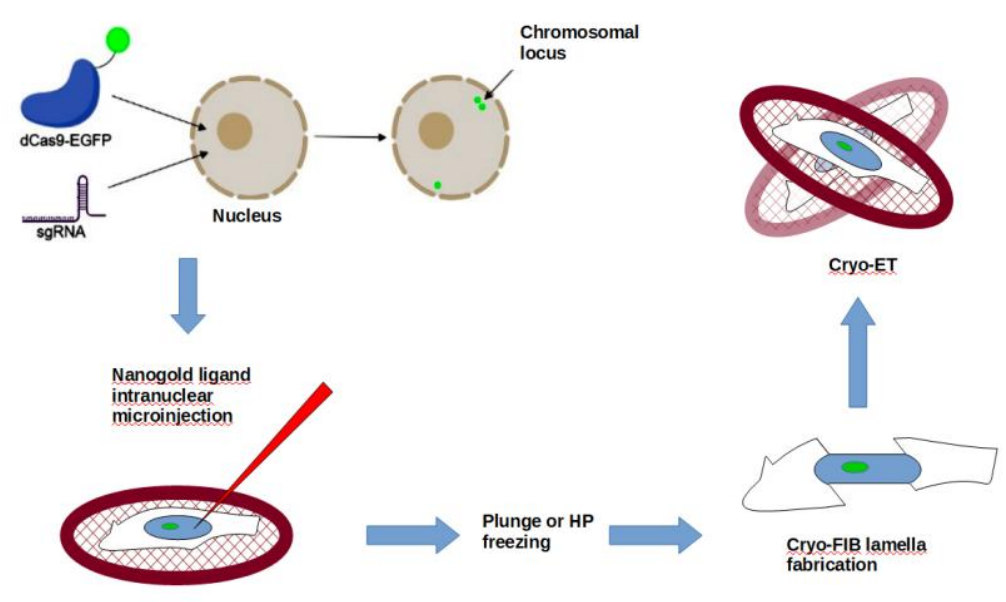

Fig. 1. Flowchart of in vivo Nanogold labeling of endogenous chromosomal loci for cryo-ET. 
Results: Our preliminary data demonstrated feasibility of above-mentioned approach with a set of DNA-binding tools (LacO-lac-rep system, TALEs, dCas) tagged with GFP, 6His and subsequent detection with anti-GFP antibodies, GBP, or Ni-NTA, conjugated with 1.4-nm gold. The passage to the cryo-ET would require further development of robust 3D-CLEM tools for location of target loci and lamella preparation with cryo-FIB.

Conclusion: A combination of nondestructive in vivo labeling of endogenous chromosomal loci with correlative cryo-FIB - cryo-ET would open new perspectives to our efforts to understand how genome is organized and functions in living cells.

Key Words: chromatin $\bullet$ in vivo gold labeling $•$ cryo-ET

Sources of Funding: This work was supported by the Russian Science Foundation (Grant \#17-15-0129) and RFBR (grant \#19-015-00273)

International Journal of Biomedicine. 2019;9 Suppl 1: S8. doi: 10.21103/IJBM.9.Suppl_1.OR6

C2019 International Medical Research and Development Corporation 